\title{
Research and Design of Fixed-Pitch Non-Grid-Connected Wind Power System
}

\author{
Ma Yundong ${ }^{1}$, Wang Junqi ${ }^{1}$ Yang Hong ${ }^{2}$ and Hu Zurong ${ }^{1}$ \\ ${ }^{1}$ Jiangsu Key Laboratory of New Energy Generation and Power Conversion \\ Nanjing University of Aeronautics \& Astronautics \\ 29 Yudao St., Nanjing 210016, China \\ Fax number: 025-84892500, e-mail: kyleo@ nuaa.edu.cn, Wang Junqi@nuaa.edu.cn \\ ${ }^{2}$ Nanjing Panda Power Technology Co., Ltd. \\ 301 East Zhongshan Road, Nanjing, China
}

\begin{abstract}
Traditional fixed-pitch wind turbine, whose rotational speed is a constant, adjusts its aerodynamic power according to the blade stall characteristic, and it has the advantage of being simple, robust and reliable, with simple and inexpensive electric systems. By introducing MPPT strategy on low wind condition and constant output power control on high wind condition, higher efficiency of fixed-pitch wind turbine can be obtained, which is realized by regulating the turbine rotational speed according to wind speed. In this paper, the variable speed regulation strategy is proposed, the stability characteristic based on the small signal model is analyzed, and the speed control technique based on disturbance observer and Internal Model PID controller is discussed in detail, some experimental results are presented, which show that the dynamic response of speed controller is favorable and efficiency of the plant is improved by tracking the maximum power point on low wind condition and keeping the turbine aerodynamic power at its rated value on high wind condition.
\end{abstract}

\section{Key words}

Wind power, Fixed-Pitch, Aerodynamic Power Regulation, Disturbance Observer, Internal Model PID

\section{Introduction}

Wind energy is a pollution-free renewable energy, which received wide attention from more and more countries around the world, and wind power plays an important part in solving the energy crisis. The total capacity of all wind turbines installed worldwide reached 175 Giga-watt in mid-2010, compared with 159 Giga-watt by the end of $2009^{[1]}$. The world market for wind turbines saw robust growth.

The rotational speed of variable speed wind turbines can be adjusted according to the change of wind speed, which allows the turbine to operate with the optimum power efficiency and highly improves the working efficiency of wind turbines. According to different wind turbine aerodynamic power regulation methods, there are two basic plant types, the fixed-pitch type and the variablepitch type. The latter one is equipped with both rotational speed and turbine blade control systems, which enable it the best power characteristic and it is has been widely used in medium and large-scaled wind farms nowadays. But for small-scaled ones, e.g. the non-grid-connected wind power system, it increases the whole costs by implying the complicated parts. Differs from pitchcontrolled types, fixed-pitch variable speed wind turbine adjusts its aerodynamic power by fully control the shaft speed and it can achieve the comparable power characteristic in theory. Besides that, it takes the advantage of being simple, reliable, and much cheaper, which makes it very suitable for medium and smallscaled wind farms.

This paper focuses on the design of generator-side converter control based on active-rectify topology fixedpitch variable speed wind power system, and the aim is to achieve the optimal control with energy efficiency criterion. The steady state and dynamic characteristics of the turbine are being researched, which show that the plant is unstable under indirect speed control strategy on high wind condition, so direct speed control strategy is introduced. Vector control is applied to PMSG, so the design method of speed controller is given, and the instability of the system is eliminated by applying the disturbance observer and Internal Model PID technique. HIL experiments are done at last, which show that the fixed-pitch variable speed wind turbine can be operated stably in all operating wind speed and the speed controller have good dynamic characteristics.

\section{Variable Speed Control Strategy Design A. Fixed-Pitch Wind Turbine Model}

Ignore the complex aerodynamic equations, fixed-pitch wind turbine model is determined by the following equations ${ }^{[2]}$ 


$$
\left\{\begin{array}{l}
P_{\mathrm{m}}=0.5 \rho \pi R^{2} v^{3} C_{\mathrm{p}}(\lambda) \\
T_{\mathrm{m}}=0.5 \rho \pi R^{3} v^{2} C_{\mathrm{T}}(\lambda)
\end{array}\right.
$$

where $T_{\mathrm{m}}$ and $P_{\mathrm{m}}$ represent the aerodynamic torque and aerodynamic power of wind turbine, $\rho$ the air density, $R$ the blade radius, $v$ the wind velocity, $\lambda$ the tip ratio speed, which is defined as: $\lambda=\omega R / v, \omega$ the rotational speed, $C_{\mathrm{p}}$ the power coefficient which is a function of $\lambda, C_{\mathrm{T}}$ the torque coefficient. A typical plot of $C_{\mathrm{p}}(\lambda)$ and $C_{\mathrm{T}}(\lambda)$ is shown in Fig. 1.

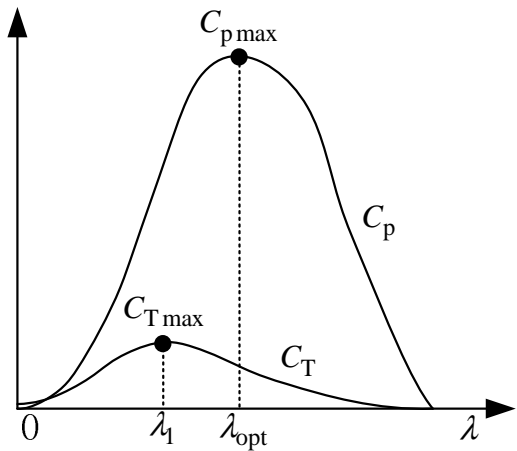

Fig. 1. $C_{\mathrm{p}}(\lambda)$ and $C_{\mathrm{T}}(\lambda)$ plot of fixed-pitch wind turbine

\section{B. Variable Speed Control Strategy}

The proposed variable speed control strategy is shown in Fig. 2, which gives the relationship between wind velocity and rotational speed reference. When the wind velocity varies between $V_{\text {start }}$ and $V_{1}$, the rotational speed of the shaft increases with the wind velocity linearly so as to keep $\lambda$ at its optimal value $\lambda_{\text {opt }}$, maximum power tracking of the wind turbine is realized. When $\omega$ equals its rated value, it is kept at this value until the turbine power reaches the maximum value, as the wind speed still increases, $\omega$ varies nonlinearly so as to keep the output power a constant value.

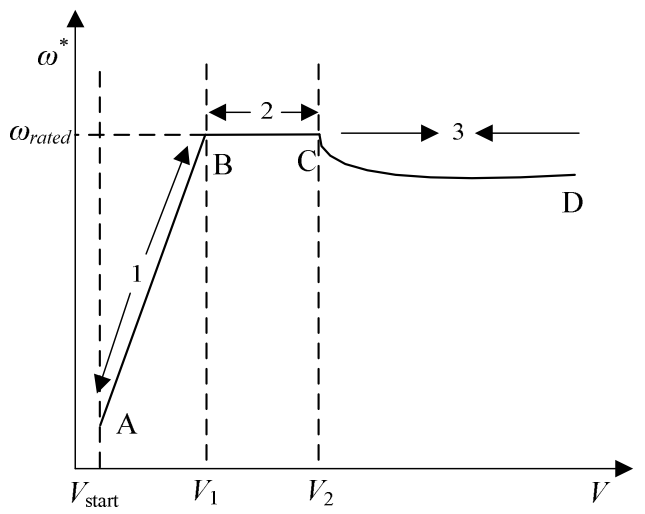

Fig. 2. Variable speed control strategy of the system

The direct speed control (DSC) diagram based on control strategy is established as Fig.3 presents. At first, speed reference $\omega^{*}$ is obtained based on the detection value of wind speed $V_{\mathrm{m}}$ by looking up the $V_{\mathrm{m}}-\omega$ table, then torque reference, which is used as reference input of generator torque control system, is obtained based on error signal between speed reference and speed feedback. At last, electromagnetic torque will follow its reference through regulating stator current regulated by generator's torque control system. The design method of the controller will be fully discussed in the following sections.

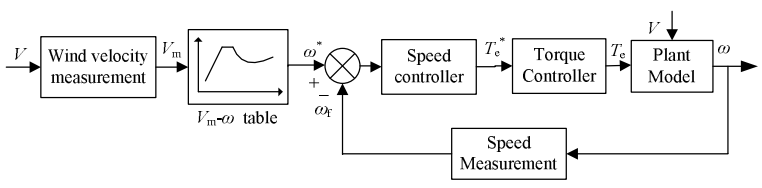

Fig. 3. Speed control diagram of the system

\section{Small Signal Modelling and Dynamic Characteristic Analysis}

The rigid drive train between wind turbine and PMSG can be modeled as

$$
T_{\mathrm{m}}-T_{\mathrm{e}}=J \frac{d \omega}{d t}+B \omega
$$

where: $J$ is turbine's moment of inertia, $\omega$ is angle velocity of generator rotor, $B$ is friction coefficient, $T_{\mathrm{m}}$ is wind turbine aerodynamic torque, $T_{\mathrm{e}}$ is generator electromagnetic torque. With a linearization method around a turbine operating point by using the first term of Taylor series, equation (2) can be expressed as

$$
J \frac{d \Delta \omega}{d t}+B \Delta \omega-\Delta T_{\mathrm{e}}=\gamma \Delta \omega+\alpha \Delta V
$$

where: $\Delta \omega, \Delta V$ are the differences of operating point $\omega_{\mathrm{op}}$, $V_{\mathrm{op}}, \quad \alpha, \gamma$ are linearization coefficients, and they can be calculated as follows ${ }^{[3]}$

$$
\begin{gathered}
\gamma=\left.\frac{\partial T_{\mathrm{m}}}{\partial \omega}\right|_{\mathrm{op}}=\frac{1}{2} \rho \pi R^{3} V_{\mathrm{op}}{ }^{2}\left(\left.\frac{d C_{\mathrm{T}}}{d \lambda} \frac{\partial \lambda}{\partial \omega}\right|_{\mathrm{op}}\right) \\
\alpha=\left.\frac{\partial T_{\mathrm{m}}}{\partial V}\right|_{\mathrm{op}}=\frac{1}{2} \rho \pi R^{3} V_{\mathrm{op}}\left[2 C_{\mathrm{T}}\left(\lambda_{\mathrm{op}}\right)-\lambda_{\mathrm{op}}\left(\left.\frac{d C_{\mathrm{T}}}{d \lambda}\right|_{\mathrm{op}}\right)\right]
\end{gathered}
$$

If $T_{\mathrm{e}}$ and $\omega$ are input and output of the system respectively, the closed loop transfer function of plant is

$$
\frac{\omega(s)}{T_{\mathrm{e}}(s)}=\frac{1}{J s+B-\gamma}
$$

As the pole of the system is $s=(\gamma-B) / J$ with $|\gamma| \square B$, the sign of the pole depends on the value of $\gamma$, which indicates the slope of $C_{\mathrm{T}}$ curve (see Fig. 1). When $\lambda$ is greater than $\lambda_{1}$, the gradient value of $C_{\mathrm{T}}$ curve is negative, so $\gamma<0$. In this region, wind turbine can operate stably without special control. When $\lambda$ is smaller than $\lambda_{1}$, the gradient value of $C_{\mathrm{T}}$ curve is positive, so $\gamma>0$. Because the frictional coefficient $B$ is very small, the system has the right half plane pole, it is unstable.

Suppose the output power of the generator is constant, the relationship curve between $T_{\mathrm{e}}$ and $\omega$ is a hyperbola. Suppose the initial intersection of $T_{\mathrm{e}}$ and $T_{\mathrm{m}}$ lies in the right part of $T_{\mathrm{m}}$ curve, which is the point $\mathrm{N}$, just as Fig. 4 presents. When the rotational speed decreases because of external perturbation, operating point of $T_{\mathrm{m}}$ and $T_{\mathrm{e}}$ move to $\mathrm{N}_{1}$ and $\mathrm{N}_{2}$ respectively, then $T_{\mathrm{m}}$ is greater than $T_{\mathrm{e}}$, which in turn drives rotational speed to increase, so operating point goes back to initial point $\mathrm{N}$ at last. A conclusion can be drawn that the right part of $T_{\mathrm{m}}$ curve is stable region as perturbation doesn't change the final position of operating point. Consider the operating point is $\mathrm{M}$, which lies in the left part of $T_{\mathrm{m}}$ curve, when the 
rotational speed increases because of external perturbation, operating point of $T_{\mathrm{m}}$ and $T_{\mathrm{e}}$ move to $\mathrm{M}_{1}$ and $\mathrm{M}_{2}$ respectively, then $T_{\mathrm{m}}$ is greater than $T_{\mathrm{e}}$, which accelerates the rotational speed further, that means the operating point will not go back to initial point M. Proper control strategy must be applied to plant when the operating point lies in the stall region in order to keep the plant a stable system.

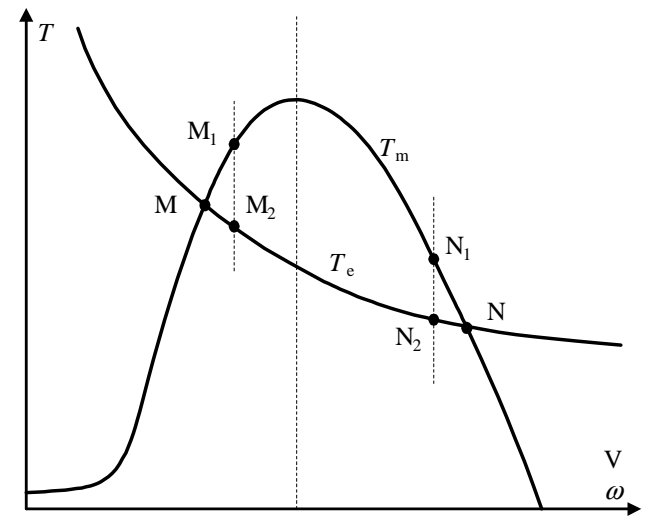

Fig. 4. Aerodynamic torque of wind turbine and generator electromagnetic torque plot

\section{Speed Controller Design}

\section{A. Speed Controller Based on Disturbance Observer}

According to the analysis of section 3 , the instability of the system is mainly reflected in the right half plane pole, and if the open-loop gain is high enough, the right half plane pole can be avoided in theory, however, the high openloop gain itself may give rise to instability of system. Meanwhile, system may be interfered greatly by highfrequency noise. In this section, the use of the disturbance observer $(\mathrm{DOB})^{[4]}$ for aerodynamic torque estimation and compensation is presented, which is used to compensate the effect of a positive $\gamma$, and make the system a stable linear system. Control diagram based on DOB is shown as follow

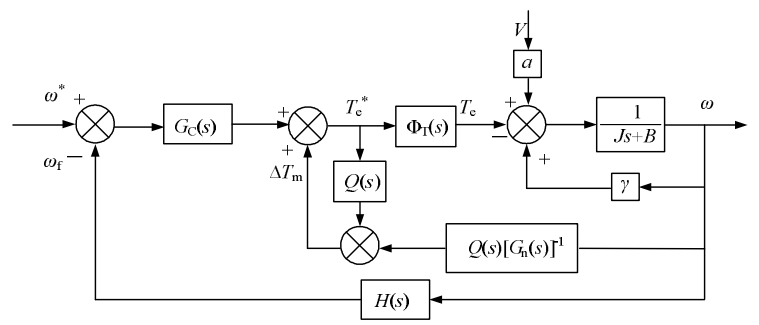

Fig. 5. Speed controller based on DOB

The equivalent simplified control diagram is

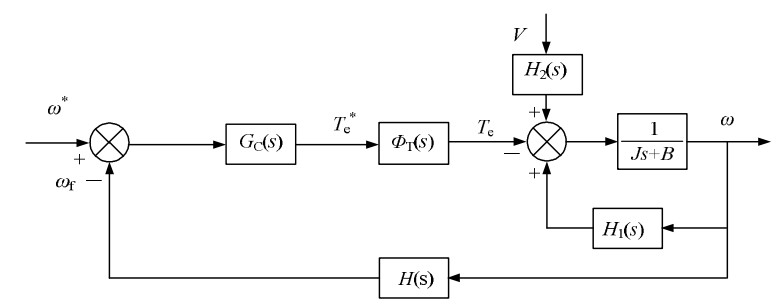

Where

$$
\left\{\begin{array}{l}
H_{1}(s) \approx \gamma \frac{\left(3 T_{\mathrm{s}}+T_{\mathrm{f}}\right) s}{\left(3 T_{\mathrm{s}}+T_{\mathrm{f}}\right) s+1}=\gamma \frac{T_{\Sigma 1} s}{T_{\Sigma 1} s+1} \\
H_{2}(s) \approx \alpha \frac{3 T_{\mathrm{s}} s}{3 T_{\mathrm{s}} s+1}=\alpha \frac{T_{\Sigma 2} s}{T_{\Sigma 2} s+1}
\end{array}\right.
$$

Then the transfer function is

$$
\begin{aligned}
\frac{\omega(s)}{T_{\mathrm{e}}(s)} & =\frac{G_{1}(s)}{1-G_{1}(s) H_{1}(s)}=\frac{\frac{1}{J_{s}+B}}{1-\frac{1}{J s+B} \gamma \frac{T_{\Sigma 1} s}{T_{\Sigma 1} s+1}} \\
& =\frac{1}{J T_{\Sigma 1} s^{2}+\left[J-(\gamma-B) T_{\Sigma 1}\right] s+B}
\end{aligned}
$$

And the necessary and sufficient condition to ensure the stability of the system is

$$
T_{\Sigma 1}=3 T_{\mathrm{s}}+T_{\mathrm{f}}<\frac{J}{\gamma-B}
$$

The physical meaning of the above equation is that: in order to ensure the turbine work steadily, time constant $T_{\Sigma 1}$ must meet the following conditions, namely, the feedback of rotational speed and the response speed of electromagnetic torque must fast enough to ensure the stability of the plant when its operating point lies in stall region. Obviously, turbine with large $\gamma_{\max }$ and $J$, requires faster rotational speed feedback speed and faster generator electromagnetic torque closed-loop response speed.

Based on the compensation of DOB, the unstable factors in the system are eliminated and the system becomes a stable linear one. So the open-loop transfer function of the system becomes

$$
\begin{aligned}
& G(s)=G_{\mathrm{C}}(s) \Phi_{\mathrm{T}}(s) \frac{1}{J_{s}+B} H(s) \\
= & G_{\mathrm{C}}(s) \frac{1}{3 T_{\mathrm{s}} s+1} \frac{1}{J s+B} \frac{1}{T_{\mathrm{f}} s+1}
\end{aligned}
$$

As the speed control loop need fast tracking ability, the speed controller parameters is tuned to meet the typical I system requirement. The transfer function of speed controller is

$$
G_{\mathrm{C}}(s)=\frac{K_{\text {ip }}(\tau s+1)}{s}
$$

where $\tau=J / B, K_{\text {ip }}=B / 6 T_{\text {s }}$.

\section{B. Speed Controller Based on Internal Model PID}

The Internal Model Control (IMC) ${ }^{[5]}$ philosophy relies on the Internal Model Principle, which states that: control can be achieved only if the control system encapsulates, either implicitly or explicitly, some representation of the process to be controlled. In particular, if the control scheme has been developed based on an exact model of the process, then perfect control is theoretically possible. In practice, process-model mismatch is common and system is often affected by unknown disturbances. The general structure of IMC depicted in Fig. 7 has the potential to achieve perfect control. 


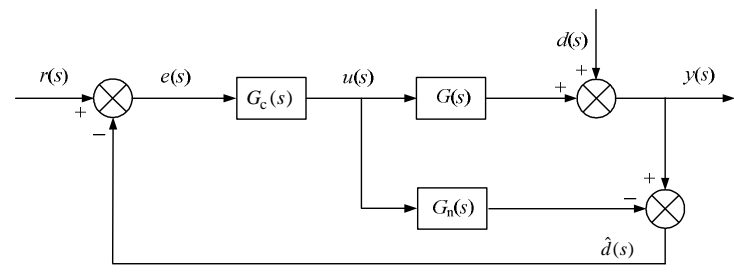

Fig. 7 Schematic of IMC scheme

In the diagram, $d(\mathrm{~s})$ is an unknown disturbance affecting the system. The manipulated input $u(\mathrm{~s})$ is introduced to both the process and its model. The process output, $y(\mathrm{~s})$, is compared with the output of the model, resulting in a signal $\hat{d}(s)$. The closed loop transfer function for the IMC scheme is

$$
Y(s)=\frac{G_{\mathrm{c}}(s) G(s) r(s)+\left[1-G_{\mathrm{c}}(s) G_{\mathrm{n}}(s)\right] d(s)}{1+G_{\mathrm{c}}(s)\left[G(s)-G_{\mathrm{n}}(s)\right]}
$$

From this closed loop expression, we can see that if $G_{\mathrm{c}}(s)=G_{\mathrm{n}}(s)^{-1}$, and if $G(s)=G_{\mathrm{n}}(s)$, then perfect set point tracking and disturbance rejection is achieved. Theoretically, even if $G(s) \neq G_{\mathrm{n}}(s)$, perfect disturbance rejection can still be realized provided $G_{\mathrm{c}}(s)=G_{\mathrm{n}}(s)^{-1}$.

The control diagram of fixed-pitch variable speed wind power system, which is based on the above internal model controller design method is shown as follow.

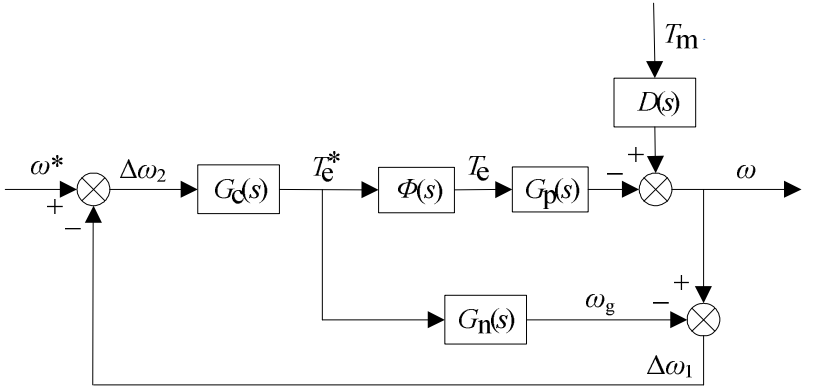

Fig. 8. IMC diagram of variable-speed wind power system

In the diagram

$$
\begin{gathered}
D(s)=G_{\mathrm{p}}(s)=\frac{1}{J s+B} \\
\Phi(s)=\frac{1}{3 T_{\mathrm{s}} s+1} \\
G_{\mathrm{n}}(s)=\frac{1}{(J s+B)\left(3 T_{\mathrm{s}} s+1\right)} \approx \frac{1}{J s\left(3 T_{\mathrm{s}} s+1\right)}
\end{gathered}
$$

The closed loop transfer function for $\omega$ is that

$$
\begin{aligned}
& \omega(s)=\frac{G_{\mathrm{c}}(s) G_{\mathrm{p}}(s) \Phi(s)}{1+G_{\mathrm{c}}(s)\left[G_{\mathrm{p}}(s) \Phi(s)-G_{\mathrm{n}}(s)\right]} \omega^{*}+ \\
& \frac{1-G_{\mathrm{c}}(s) G_{\mathrm{p}}(s) \Phi(s)}{1+G_{\mathrm{c}}(s)\left[G_{\mathrm{p}}(s) \Phi(s)-G_{\mathrm{n}}(s)\right]} D(s) T_{\mathrm{m}}(s)
\end{aligned}
$$

It is obvious that if $G_{\mathrm{c}}(s)=G_{\mathrm{p}}(s) \Phi(s)^{-1}$, the disturbance effect caused by $T_{\mathrm{m}}$ can be fully eliminated. Additionally, since discrepancies between process and model behaviour usually occur at the high frequency end of the system's frequency response, a low-pass filter $Q(s)$ is usually added to attenuate the effects of process-model mismatch. Thus, the internal model controller is usually designed as the inverse of the process model in series with a low-pass filter. In this paper, low-pass filter is selected as follow

$$
Q(s)=\frac{2 \tau s+1}{(\tau s+1)^{2}}
$$

So

$$
G_{\mathrm{c}}(s)=\left[G_{\mathrm{p}}(s) \Phi(s)^{-1}\right] Q(\mathrm{~s})=\frac{J s(2 \tau s+1)\left(3 T_{\mathrm{s}} s+1\right)}{(\tau s+1)^{2}}
$$

A equivalent diagram of Fig. 8 is shown below

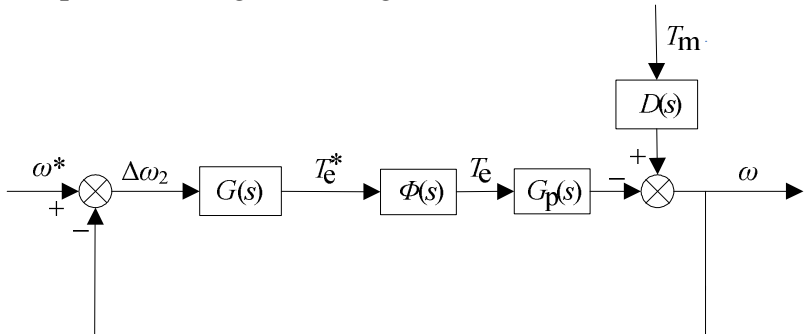

Fig. 9 Equivalent IMC control diagram of variable-speed wind power system

Where

$$
G(s)=\frac{G_{\mathrm{C}}(s)}{1-G_{\mathrm{n}}(s) G_{\mathrm{C}}(s)}=\frac{J(2 \tau s+1)\left(3 T_{\mathrm{s}} s+1\right)}{\tau^{2} s}
$$

It is obvious that $G(s)$ has the same format as conventional PID controller, and

$$
\left\{\begin{array}{l}
K_{\mathrm{p}}=\frac{J\left(2 \tau+3 / f_{\mathrm{s}}\right)}{\tau^{2}} \\
K_{\mathrm{i}}=\frac{J}{\tau^{2}} \\
K_{\mathrm{d}}=\frac{6 J}{\tau f_{\mathrm{s}}}
\end{array}\right.
$$

The speed controller $G(s)$ has only one adjustable parameter $\tau$ which determines system's dynamic performance and robustness. System's response speed will become faster as $\tau$ decreases. However, system's robustness will become poor, causing fluctuations easily. On the contrary, if $\tau$ increases, system's robustness will become fine but response speed will become slow. In a word, the desired controller can regulate its parameter online based on expectation characteristic.

\section{HIL Experimental Results}

Several experiments have been carried out to study the performance of a $10 \mathrm{~kW}$ non-grid-connected wind energy conversion system based on RT-LAB. DSP controller based on TMS320x2808 is connected with RT-LAB through the I/O port. All the signal that the controller need, for example, the rotational speed of PMSG, stator voltage, stator current are first transformed into analog signals using the DAC (OP5330) in RT-LAB, then they are transmitted to DSP controller. PWM signals produced by DSP which are used to control the virtual plant simulation model are sent to Digital In port (OP5311) of RT-LAB, the wind generator controllers under test behave as if they were connected to a real system. Experimental parameters are as follows:

- Rated power: $10 \mathrm{~kW}$

- DC bus voltage: 300VDC

- Rated rotational speed: $152.7 \mathrm{rpm}(16 \mathrm{rad} / \mathrm{s})$

- Rated wind speed: $10.5 \mathrm{~m} / \mathrm{s}$ 
- Maximum wind turbine power coefficient: 0.364

- Maximum wind turbine torque coefficient: 0.0485

- Radius of wind turbine: $4 \mathrm{~m}$

- Moment of inertia: $8 \mathrm{~kg} \cdot \mathrm{m}^{2}$

- Frictional coefficient: $0.05 \mathrm{~N} \cdot \mathrm{m} \cdot \mathrm{s}$

- Pole pairs of PMSG: 8

- Inductor of stator: $5 \mathrm{mH}$

- Resistor of stator: $0.1 \Omega$
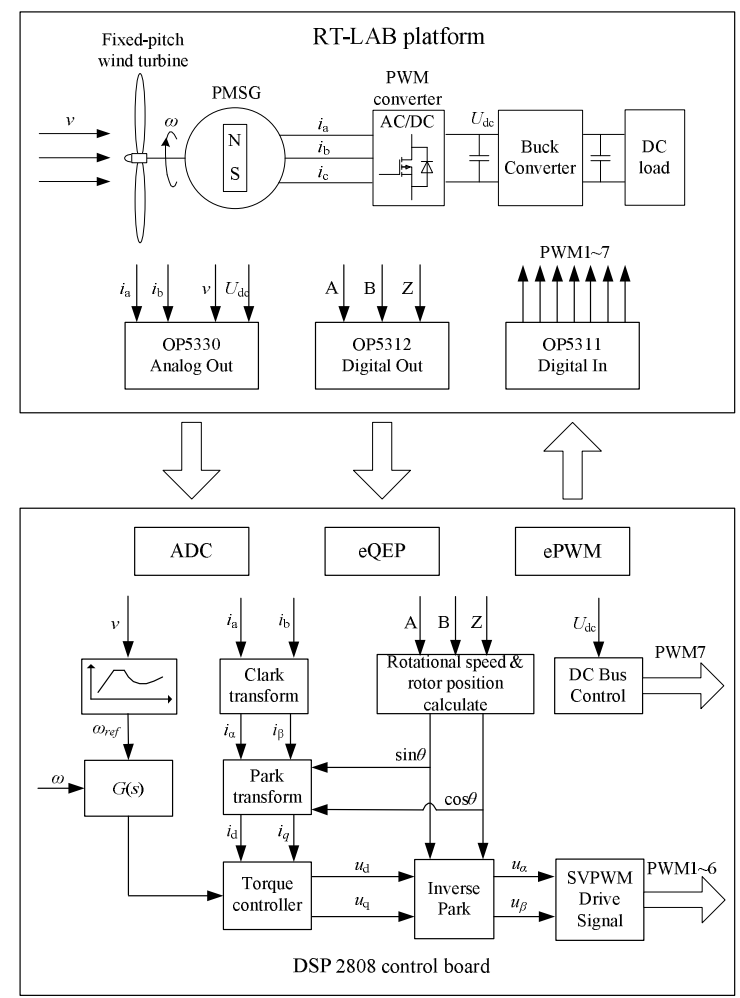

Fig. 10 HIL platform based on RT-LAB

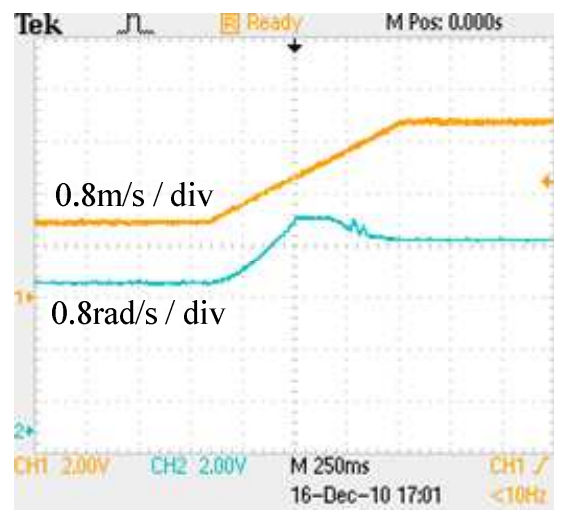

Fig. 11(a) $v$ VS. $\omega$

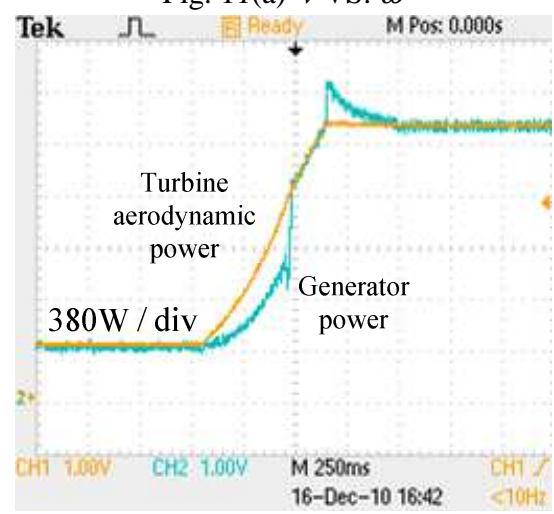

Fig. 11(b) turbine aerodynamic power VS. generator power

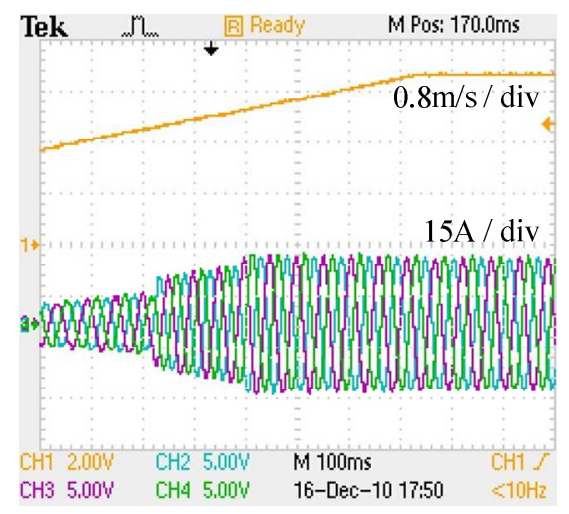

Fig. 11(c) $v$ VS. stator current

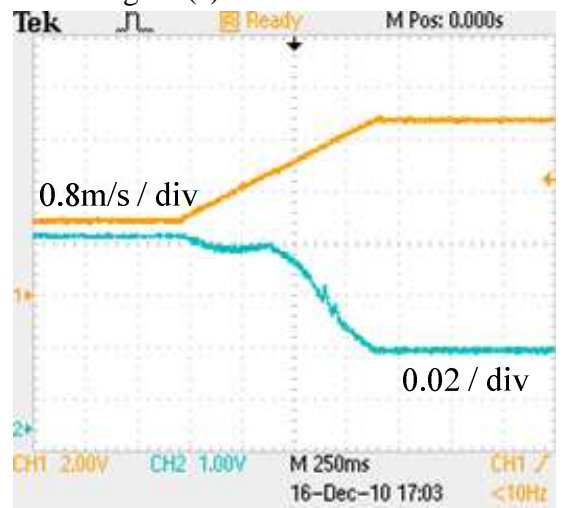

Fig. 11(d) $v$ VS. $C_{\mathrm{p}}$

Fig. 11 shows the response to a ramp increase in wind velocity from $5.5 \mathrm{~m} / \mathrm{s}$ to $13 \mathrm{~m} / \mathrm{s}$. The shaft rotational speed curve (Fig. 11(a)) can be divided into three parts: when $\omega$ doesn't reach its rated value $16 \mathrm{rad} / \mathrm{s}$, it increases with the wind velocity linearly so as to keep $C_{\mathrm{p}}$ at its maximum value $C_{\text {pmax }}$, maximum power tracking of the wind turbine is realized as Fig. 8(b) presents. When $\omega$ equals $16 \mathrm{rad} / \mathrm{s}$, it is kept at this value until the turbine power reaches the rated value of $10 \mathrm{~kW}$, as the wind speed still increases, the current and electromagnetic torque increase to decelerate the PMSG into the stall region, so as to keep the output power at its rated value. $C_{\mathrm{p}}$ value drops from 0.365 to 0.15 as Fig. 11(d) shows.

\section{Conclusion}

Because of the influences of the non-linear relation among the aerodynamic torque, rotational speed and wind speed, in high wind speed, the speed system of fixed-pitch variable speed wind turbines has already turned into an unstable non-linear system. So the use of disturbance observer and Internal Model PID Controller for design of speed control system is presented. Experiments are done and indicate that fixed-pitch variable speed wind turbines can be operated not only for MPPT in low wind speed but also for constant power control in high wind speed.

\section{References}

[1] World Wind Energy Report 2009. WWEA. March 2010

[2] Iulian Munteanu, Antoneta Luliana Bartcu, Nicolaos Antonio Cutululis, et al. Optimal Control of Wind Energy System. Springer London, 2008.

[3] B. Neammanee, K. Krajangpan, S. Sirisumrannukul, et al. Maximum Peak Power Tracking-Based Control Algorithms with Stall Regulation for Optimal Wind Energy Capture, 
Power Conversion Conference, Nagoya, April 2007: $1424 \sim 1430$.

[4] Hu Zurong, Wang Junqi, Ma Yundong. Research on speed control system for fixed-pitch wind turbine based on disturbance observer, WNWEC 2009, Nanjing China, Sep. 2009: 221 225.

[5] S. B. Alabi, O. Taiwo. Internal Model Control of Uncertain Systems: An Improved Approach. ACSE 05 Conference, CICC, Cairo, Egypt, 19-21 Dec. 2005: 161 166. 